\title{
Osteoprotegerin gene-modified BMSCs with hydroxyapatite scaffold for treating critical-sized mandibular defects in ovariectomized osteoporotic rats
}

\author{
Xian Liu ${ }^{1}$, Chongyun Bao ${ }^{1}$, Hockin H.K. Xu ${ }^{2-5}$, Jian $\operatorname{Pan}^{1}$, Jing Hu${ }^{1}$, \\ Ping Wang ${ }^{1,2}$, En Luo $^{1}$
}

${ }^{1}$ State Key Laboratory of Oral Diseases, West China Hospital of Stomatology, Sichuan University, Chengdu, Sichuan 610041, China

${ }^{2}$ Biomaterials \& Tissue Engineering Division, Department of Endodontics, Prosthodontics and Operative Dentistry, University of Maryland Dental School, Baltimore, MD 21201, USA

${ }^{3}$ Center for Stem Cell Biology and Regenerative Medicine, University of Maryland School of Medicine, Baltimore, MD 21201, USA

${ }^{4}$ Marlene and Stewart Greenebaum Cancer Center, University of Maryland School of Medicine, Baltimore, MD 21201, USA

${ }^{5}$ Mechanical Engineering Department, University of Maryland, Baltimore County, MD 21250, USA

\section{Correspondence:}

Dr. Ping Wang, University of Maryland Dental School, 650 W. Baltimore St, Baltimore, MD 21201 (Email: pwang@umaryland.edu; Tel: +1-443-676-6088). Dr. En Luo, Professor, West China Hospital of Stomatology, Sichuan University, China (Email: luoen521125@sina.com).

Running Head: Osteoprotegerin-modified BMSCs for bone repair in ovariectomized osteoporotic rats. 


\section{Abstract}

Women with postmenopausal osteoporosis are at a high risk for fracture as their bone resorption rate exceeds bone formation rate, resulting in decreased bone mineral density and microarchitectural deterioration. Osteoprotegerin (OPG), a known therapeutic agent capable of inhibiting osteoclastogenesis, has been used in treatment of chronic bone resorptive diseases. On the other hand, bone mesenchymal stem cells (BMSCs) play an important role in bone formation. To inhibit excessive bone resorption and increase bone formation, we developed a novel therapeutic strategy by genetically modifying BMSCs for OPG delivery. The OPG gene-modified BMSCs were seeded on hydroxyapatite (HA) scaffolds to promote bone regeneration in critical-sized mandibular bone defects in ovariectomy (OVX) induced osteoporotic rats. Rat BMSCs were infected with human OPG adenoviruses (OPG-BMSCs). The gene-modified cells expressed higher OPG gene level than the control Ad-BMSCs $(p<0.05)$ and maintained high expression of OPG protein for more than 2 weeks. Our in vitro bone resorption experiment demonstrated that OPG-BMSCs were capable to suppress osteoclast differentiation and subsequently inhibit osteoclast-mediated bone resorption. The micro-CT and histological results showed that HA-OPG-BMSC constructs boosted bone formation and reduced osteoclastogenesis in OVX rat mandibular bone defects. In conclusion, the novel OPG-BMSC-HA constructs were demonstrated to be able to orchestrate bone-forming BMSCs and bone-resorbing osteoclasts, with the potential for osteoporotic-related bone defect reconstruction applications.

Key words: Osteoprotegerin, BMSCs, Bone regeneration, Ovariectomy, Gene therapy. 


\section{Introduction}

Postmenopausal osteoporosis is a common debilitating systematic disease, characterized by gradually reduced bone mineral density and compromised bone strength [1]. This skeletal disorder poses a significant threat to millions of postmenopausal women for osteoporotic fractures and associated complications, and brings difficulties for subsequent reconstruction of bone defects. The receptor activator of nuclear factor kappa B (RANK) / RANK ligand (RANKL) / osteoprotegerin (OPG) system has been known to play a central role in bone remodeling. Dysregulation of the RANK/RANKL/OPG system has been implicated in the pathophysiology of disorders of mineral metabolism, such as postmenopausal osteoporosis [2]. The RANK, produced by osteoblast lineage cells, periodontal ligament cells and $\mathrm{T}$ lymphocytes, can lead to osteoclastogenesis via interaction with RANKL on the surface of osteoclast precursors. This process can be potently inhibited by OPG, which has been known as a negative regulator of osteoclast formation, function and survival by binding with RANK [3]. OPG was first identified in 1997 [4]. Simonet et al. reported that transgenic mice expressing OPG exhibited a generalized increase in bone density and a decrease in osteoclast cell number [4]. Administration of recombinant OPG produced the same effects in normal mice to transgenic mice and recombinant OPG administration could prevent bone loss in ovariectomized (OVX) mice [4]. In vitro study showed that OPG blocked osteoclastogenesis in a dose-dependent manner [4]. Based on the anti-osteoclast function, OPG has been used for multiple bone remolding disorders associated with excessive osteoclast activity [5-10]. Capparelli et al. reported that even a single bolus intravenous injection of OPG resulted in a 
rapid (within 12 hours) and profound (up to 95\%) reduction in the percentage of bone surfaces occupied by osteoclasts at 5 and 10 days in a rat animal model [9]. Although OPG can be delivered via protein injection, osteoporosis is a chronic disease that requires decades of therapy and thus repetitive injections.

Gene therapy has the potential to reach long-term therapeutic effects by delivering genes of anti-resorptive proteins to patients, circumventing the need of repeated administration. Plasmid and virus mediated OPG expressions have been proven to be effective in inhibiting osteoclastogenesis in experimental tooth movement model [6] and in ameliorating osteoporosis associated with estrogen deficiency for an extended period (up to 18 months) in OVX mice [10]. Another avenue to provide sustained treatment would be through a cell-mediated gene delivery method. Bone marrow mesenchymal stem cells (BMSCs) with multi-directional differentiation potentials are the most important cell sources for bone tissue engineering [11]. Aside from the direct therapeutic potential of serving as seeding cells, BMSCs are also widely used as a vector for gene transfer $[12,13]$. However, there have been no studies involving stem cell mediated OPG expression for bone tissue engineering.

The objectives of the present study were to genetically modify BMSCs for OPG overexpression, and investigate the anti-osteoclastogenic effect of OPG gene-modified BMSCs in vitro via bone resorption experiment and in vivo in a critical sized mandibular bone defect model in OVX induced osteoporotic rats for the first time. The following hypotheses were tested: (1) rat BMSCs can be successfully modified genetically to have OPG overexpression; (2) OPG gene-modified BMSCs can inhibit osteoclastogenesis in vitro; (3) 
OPG gene modification of BMSCs will not have adverse effects on cell attachment and proliferation on hydroxyapatite (HA) scaffolds; (4) OPG gene-modified BMSCs will exert anti-osteoclastogenic effects and promote bone regeneration in OVX rats.

\section{Material and methods}

\subsection{Isolation and culture of rat BMSCs}

BMSCs were obtained from 7-day Sprague-Dawley (SD) rats (experimental animal center of Sichuan University), following the procedure that we previously reported [14]. Briefly, BMSCs were collected by flushing bone marrow out of the rats' femurs and tibias using Dulbecco's modified Eagle's medium (Gibco BRL, Gaithersburg, MD) supplemented with 10\% fetal bovine serum (Gibco BRL) and 100 units/ml penicillin-streptomycin (Gibco BRL). Cells were cultured in a $37^{\circ} \mathrm{C}, 5 \% \mathrm{CO}_{2}$ incubator for 48 hours to allow initial cell adherence to culture flasks. Upon $80 \%$ to $90 \%$ confluence, cells were passaged and purified. Passage 3 cells were used for gene transfection and subsequent experiments.

\subsection{Gene transfection of rat BMSCs}

Serotype 5 recombinant replication-defective human E3 ${ }^{-}$adenoviruses that encode OPG/enhanced green fluorescent protein (Ad5-OPG) sequence were constructed by Vector Gene Technology Company Ltd., China. The recombinant adenovirus transfer vector sequences information was shown in supplementary Fig. S1. The gene was driven by a mouse cytomegalovirus promoter. Both human OPG cDNA segment and enhanced green 
fluorescent protein (EGFP) cDNA segment were contained in this adenoviral reconstruction. Cells transfected with Ad5-OPG were referred to as OPG-BMSCs. Ad5-EGFP was used as the control vector. Cells transfected with Ad5-EGFP were referred to as Ad-BMSCs. The non-transfected BMSCs were referred to as BMSCs. $2 \times 10^{5}$ BMSCs were seeded in each well of 6-well plates. When BMSCs reached 60\%-80\% confluence, cells were exposed to 80 multiplicity of infection (MOI, pfu/cell) for 3 days. Transduction efficiency was assessed by counting EGDP-positive cells among the total cell count observed after 3 days of culture under fluorescent microscopy (Leica, German). Six samples with one randomly-selected field from each sample at $10 \times$ magnification were counted via Image $J$ software to get the total cell number and EGFP-positive cell number, respectively.

\subsection{OPG gene expression and protein secretion}

Quantitative real-time reverse transcription polymerase chain reaction was used to measure the expression of OPG mRNA in the transfected cells. Relative expression was evaluated using the $2^{-\Delta \Delta C t}$ method [15]. Briefly, total cellular RNA was isolated from OPG-BMSCs at day 1, 3, 5, 7, 14 and cDNA were prepared according to the manufacturer's instruction of PrimeScript RT reagent kit (Takara Bio, Shiga, Japan). The sequences of human specific primers were synthesized commercially (Shengong Co., Shanghai, China), and the polymerase chain reaction were forward 5'-GGCAACACAGCTCACAAGAA-3', reverse 5'-CTGGGTTTGCATGCCTTTAT-3' encoding the OPG gene and forward 5'-AGACAGCCGCATCTTCTTGT-3', reverse 5'-CTTGCCGTGGGTAGAGTCAT-3' 
encoding the sequence of glyceraldehyde-3-phosphate dehydrogenase (GAPDH) gene. The Ad-BMSCs at each time points were used as the control. The $\mathrm{C}_{\mathrm{t}}$ values of relative expression of OPG-BMSCs at 1 day served as the calibrator. The $\mathrm{C}_{\mathrm{t}}$ values of hOPG genes at different days were normalized by the $C_{t}$ values of the human housekeeping gene GAPDH to obtain the $\Delta \mathrm{C}_{\mathrm{t}}$ values.

The expression of OPG protein was detected by Western-blot. OPG-BMSCs, Ad-BMSCs and BMSCs were seeded at a density of $2 \times 10^{5}$ cells per well in 6-well plates. Cells were lysed and protein was collected at 14 days. $40 \mu \mathrm{g}$ of total protein was analyzed on a 10\% SDS-polyacrylamide gel and electro-transferred to a nitrocellulose membrane (Bio-Rad, Hercules, USA). The OPG antibody used in the Western-blot was a goat polyclonal IgG antibody (sc-8468, Santa Cruz, CA, USA) from Santa Cruz Biotechnology, which was recommended for OPG detection of mouse, rat and human origins. Subsequently, a Horseradish peroxidase-conjugated chicken anti-goat IgG secondary antibody (sc-516086, Santa Cruz) was applied. The membrane was visualized by the enhanced chemiluminescence detection system (ECL, Amersham Pharmacia Biotech, Piscataway, NJ, USA) following the manufacturer's instruction.

The OPG protein secreted from cells was evaluated by measuring OPG amount in the conditioned medium via Osteoprotegerin Human SimpleStep ELISA ${ }^{\mathrm{TM}}$ (Thermo Electron, Waltham, MA, USA). We followed the method of a previous report [15]. Briefly, cells were cultured in 6-well plates with $2.5 \mathrm{~mL}$ medium and the cell supernatants were collected following a 24-hour serum-free culture at day 1, 3, 5, 7 and 14. Cells were then trypsinized 
and the cell number was determined using a hemocytometer counting chamber (Lumicyte, Propper Manufacturing Company, Long Island, NY). The supernatants were centrifuged at $3000 \mathrm{rcf}$ and $4{ }^{\circ} \mathrm{C}$ for $10 \mathrm{~min}$ to remove cellular debris and particulates and stored in aliquots $80{ }^{\circ} \mathrm{C}$. OPG concentration was measured according to the manufacturer's instructions. The secreted OPG amount was reported as ng/day/million cells.

\subsection{Osteogenic differentiation of OPG-BMSCs}

The osteogenic ability of transfected cells was detected by Alizarin Red Staining (ARS) after 2 weeks of osteogenic differentiation. The cells were cultured in a 12 -well plate in the osteogenic medium (DMEM containing 10\% FBS, $100 \mathrm{mM}$ dexamethasone, $10 \mathrm{nM}$ $\beta$-glycerophosphate, and $0.25 \mathrm{mM} \mathrm{L}$-ascorbic acids). Two weeks later, cells were fixed in $10 \%$ formaldehyde and stained with ARS for 1 hour to visualize the presence of calcified deposition produced by cells.

\subsection{In vitro bone resorption experiment}

In vitro bone resorption experiment was taken to test the bone-protective function of OPG secreted from OPG-BMSCs. Osteoclast precursor Raw264.7 cells (ATCC, Manassas, VA, USA) were seeded on cortical bone wafers $(6 \mathrm{~mm} \times 6 \mathrm{~mm} \times 0.3 \mathrm{~mm}$; sections were obtained by cutting from purchased bovine femur, $\mathrm{n}=8$ ) at a density of $1 \times 10^{4} / \mathrm{cm}^{2}$ and cultured for 24 hours in the medium of á-MEM supplemented with 10\% FBS (Gibco BRL, MD, USA). The Raw264.7 cell seeded cortical bone wafers were then transferred to 6-well 
plates which were pre-seeded with either OPG-BMSCs or Ad-BMSCs at a density of $1 \times 10^{4}$ / $\mathrm{cm}^{2}$ for 1 day. The cortical bone samples were suspended in culture medium. Raw264.7 cells can be differentiated into osteoclast-like cells in the presence of Macrophage Colony-stimulating Factor (M-CSF) and RANKL [16, 17]. Cells were serum-starved overnight, then treated with M-CSF (40ng/ ml) and Murine-soluble RANKL (40ng/ ml) (Peprotech, NJ, USA) in á-MEM supplemented with 1\% FBS. The culture medium contained M-CSF (40ng/ ml) and Murine-soluble RANKL (40ng/ ml) was changed every three days. After 2 weeks, half of the wafers $(n=4)$ were dried and sputter-coated with gold for Scanning Electron Microscope (KYKY-2800). To measure the areas of resorption lacunae on the wafers, the other samples $(n=4)$ were cleaned with trypsin-EDTA solution first and then scraped with domestic bleach to clear the cells from the surface [18]. Images of wafers were taken with a Minolta Dimage Scan Dual (Minolta, Ramsey, NJ, USA) and the enclosed area on the surface of wafers was analyzed using Osteometrics ${ }^{\mathrm{R}}$ software (OsteoMetrics, Atlanta, GA, USA). It was measured by viewing 8 fields for each group (four samples per group, with two randomly selected fields per sample, $\mathrm{n}=8$ ). Two observers independently performed the analysis. The average results from the two observers were used for the final calculation. Percentage of resorbed area was measured as the ratio of the resorbed area to the total area in the acquired images.

\subsection{Preparation of BMSC-seeded HA scaffolds}

The HA scaffolds were prepared by Sichuan University Research Center for 
Nano-Biomaterials as discs $(\phi 5 \mathrm{~mm} \times 1.0 \mathrm{~mm})$ with a porosity of $60 \%$ and an average pore size of $300 \mu \mathrm{m} \pm 50 \mu \mathrm{m}$. After being autoclaved, the HA discs were bathed in $\alpha$-MEM culture medium for 24 hours before cell seeding. BMSCs were seeded at a density of $2 \times 10^{5} / \mathrm{cm}^{2}$ on the surface of HA discs. After a period of 4-hour incubation, additional culture media were added.

For the in vitro study, the Ad5-OPG was added into the medium at the time of cell seeding on the discs. Samples were harvested at 7 days for Electron Microscope (KYKY-2800) scanning and at 1, 2, 3, 4, 5, 6, and 7 days for 3-(4, 5-dimethylthiazol-2-yl)-2, 5-diphenyltetrazolium bromide (MTT) tests. The MTT absorbance at a wavelength of 570 nm was measured using a Beckman DU7400 spectrophotometer.

\subsection{Animal surgery}

The protocol of the animal experiment was approved by the Animal Care Committee of Sichuan University. All the Sprague-Dawley rats underwent bilateral ovariectomization to establish the OVX induced osteoporosis model following our previous report [19]. After 3 months, bilateral critical sized bone defects were created in the rat mandibles. Briefly, under general anesthesia, a 1.0 to $1.5 \mathrm{~cm}$ sagittal incision was made along the lower edge of mandible body to expose the bone. A full-thickness $5 \mathrm{~mm}$ diameter round bone defect was made on each side of the mandible [20]. The reconstruction of the defects was randomly assigned into 3 groups: 1) HA control group; 2) BMSCs/HA group: BMSCs were seeded at a density of $2 \times 10^{5} / \mathrm{cm}^{2}$ and cultured on HA for 6 days before in vivo implantation; 3 ) 
OPG-BMSCs/HA group: OPG-BMSCs were seeded on HA at a density of $2 \times 10^{5} / \mathrm{cm}^{2}$. The Ad5-OPG transfection was performed at day 3 when the cell confluence was approximately at $80 \%$, and cultured for another 3 days before in vivo implantation. The periosteum was removed during the surgery and the incision was closed in layers using 4-0 resorbable sutures. A total of thirty-six Sprague-Dawley rats (180 g to $220 \mathrm{~g}$ ) were used in this study. Three groups and three time points (4, 6 and 8 weeks) were designed for tests. Each group has twelve rats with four rats and eight defects for each time point.

\subsection{Microcomputed tomography (micro-CT) scanning}

The rats were sacrificed at 4, 6 and 8 weeks and mandibles were harvested for micro-CT scanning (Scanco Medical, Bassersdorf, Switzerland). Four samples per group were analyzed for each time point $(\mathrm{n}=4)$. The system of micro-CT was set to $70 \mathrm{kV}, 114 \mathrm{~mA}$, $500 \mathrm{~ms}$ integration time, and the scanning region had a resolution of $2048 \times 2048$ pixels and an isotropic voxel size of $10 \mu \mathrm{m}$ for detail evaluation. A threshold value of $205 \mathrm{mg} \mathrm{HA} / \mathrm{ccm}$ was set to distinguish mineralized tissue from the unmineralized tissue [21]. The defect of mandible was selected as the volume of interest. The mineral area volume to total volume ratio (MV/TV) was calculated to indicate the portion of highly mineralized area, including HA and mineralized bone. The bone mineral density (BMD) was calculated to compare the difference of mineralization in defects among three groups.

\subsection{Histology examination}


Samples were fixed in $4 \%$ paraformaldehyde solution for 3 days, dehydrated with alcohol and embedded in methylmethacrylate without decalcification. The cutting surface was sectioned longitudinally along the axial plane and the samples were continuously cut until reaching the areas where both the host bone and scaffolds were exposed. The sections of $100 \mu \mathrm{m}$ thickness were performed using a model SP1600 microtome (Leica Microsystems, Wetzlar, Germany), then polished to approximately $15 \mu \mathrm{m} \sim 20 \mu \mathrm{m}$. The sections from each sample were stained with Masson's Trichrome. New bone area and defect area was measured within the boundaries of the defect in each section by Image Pro 5.0 Software (Media Cybernetics, Carlsbad, CA). New bone area fraction was calculated as new bone area divided by defect area. This was measured by viewing 6 fields of each group $(n=6)$.

The Tartrate resistant acid phosphatase (TRAP) staining was performed via a commercial kit (Sigma-Aldrich, St. Louis, MO). The TRAP staining positive cells with three or more nuclei were identified as osteoclastic cells, and the number of osteoclasts was counted at $\mathrm{x} 40$ magnification [22]. All sections $(n=6)$ were evaluated by two independent reviewers unaware of the identity of the groups. The scores were averaged for each group to determine the overall score for the group.

Human OPG expressed in engineered bone constructs were detected using primary antibodies against human OPG (rabbit polyclonal anti-human OPG; PL0304462). Briefly, the tissue sections were deparaffinized and rehydrated before incubating for $40 \mathrm{~min}$ in citrate buffer at $70{ }^{\circ} \mathrm{C}$ for epitopes recovery. The endogenous peroxidase activity was blocked with $3 \% \mathrm{H}_{2} \mathrm{O}_{2}$. The slides were then blocked with $1 \%$ BSA for 30 min to suppress nonspecific 
staining, and stained with primary antibodies (1:100) overnight in a humidified environment. Subsequently, the secondary antibody against rabbit IgG (1:500) was used to incubate the sections for $30 \mathrm{~min}$ at $37{ }^{\circ} \mathrm{C}$. Incubation was followed by streptavidin-HRP and diaminobenzidine (DAB) substrate, and counterstaining with hematoxylin solution.

\subsection{Statistical analysis}

All data were expressed as mean $\pm \mathrm{SD}$, and the statistical analyses were performed using SPSS 12.0 (SPSS, Chicago, IL, USA). Statistical significance was analyzed by using the one-way analyses of variance (ANOVA), followed by post hoc LSD (least significant difference) tests. All statistical analysis was considered significant when $\mathrm{p}<0.05$.

\section{Results}

The Ad5-OPG transfected rat BMSCs were shown in Fig. 1. Passage 3 rat BMSCs were used for transfection. The cells were fibroblast-like, appearing polygonal or spindle-shaped with long processes (Fig. 1A). Three days after transfection, as shown in the Fig. 1B, nearly 95\% cells expressed enhanced green fluorescent protein under fluorescence microscope. The OPG-BMSCs expressed a significantly higher level of OPG protein at 14 days, compared to the Ad-BMSCs and BMSCs (Fig. 1C). The OPG mRNA expression reached the peak level at day 5 and maintained a relatively high level of expression at 14 days (Fig. 1D). The concentration of OPG secreted by OPG-BMSCs to the culture supernatant at 1, 3, 5, 7 and 14 days was shown in Fig. 1E. The OPG-BMSCs produced high OPG during the entire culture 
period, while Ad-BMSCs and BMSCs had no OPG production. The OPG concentration of OPG-BMSCs peaked at 3 days, followed by a moderate decline. After 2 weeks of osteogenic differentiation, ARS staining revealed higher and more densely stained particles in OPG-BMSCs than Ad-BMSCs and BMSCs, suggesting that OPG gene transfection promoted mineralization of the cells (Fig. 1F and Fig. S2).

Fig. 2 showed that OPG secreted by OPG-BMSCs was able to inhibit bone resorption in vitro. Raw264.7 cells (Fig. 2A) could be induced into multi-nucleolus osteoclast cells (Fig. 2B). Induced osteoclasts were cultured on the cortical bone wafer. Fewer osteoclasts and absorbed bone lacunas were observed when co-cultured with OPG-BMSCs (Fig. 2C) than the control group (Fig. 2D). The difference was statistically significant between the two groups as shown by the quantitative data in Fig. 2F. The Fig. 2E showed high magnification of osteoclasts and absorbed lacuna on the cortical bone wafer.

SEM scanning showed that both OPG-BMSCs and BMSCs attached well onto the HA scaffolds, exhibiting pseudopodia extended to the surface and pores of the scaffolds (Fig. 3A, 3B). There was no difference in cell viability and proliferative profiles between the transfected and non-transfected BMSCs seeding on top of HA scaffolds ( $p>0.05)$ (Fig. 3C). Fig. 3D showed that the cell seeded HA scaffold was implanted in the critical-sized mandibular bone defect in rats.

Typical sagittal sections of micro-CT scanning were shown in Fig. 4. The high density area was reduced with time and some relatively low mineralized area was formed in the defect of HA control group. Less resorption of density was found in BMSCs group, 
compared to HA control group. In the OPG-BMSCs seeded group, mineralized density increased with time and no obvious density reduction was found in the bone defect. The statistical analysis indicated that the MV/TV was significantly higher in OPG-BMSCs group than the other two groups at each time points $(p<0.05)$. The BMSCs group also showed significantly higher $\mathrm{MV} / \mathrm{TV}$ at 6 and 8 weeks than the control group $(p<0.05)$. The OPG-BMSCs group exhibited the highest BMD value at each time points. No significant difference in BMD was detected between BMSCs group and the control group $(p>0.05)$.

The representative histological images at 6 and 8 weeks were shown in Fig. S3, Fig. 5 and Fig. 6, respectively. At 6 weeks, higher amount of extracellular matrix, connective tissue and newly calcified bone was found in the pores of the scaffolds in the OPG-BMSCs group and BMSCs group than the control group (Fig. S4, A, C, E). The deposit of mineralized new bone increased greatly at 8 weeks as shown by the overview of the defect areas (Fig. 5) and images at a high magnification (Fig. 6). The OPG-BMSCs group exhibited more new bone than the other two groups. At high magnification, it clearly showed that osteoclast cells were appeared around the scaffold and newly formed tissue in the control and BMSCs group (Fig. S4B, D; Fig.6B, D). In contrast, few osteoclasts were identified in OPG-BMSCs group at the same magnification level (Fig. S3F; Fig. 6F). These results showed that local OPG delivered by OPG-BMSCs inhibited osteoclastogenesis.

The representative TRAP staining images at 6 weeks were shown in Fig. 7A, B and C. The red staining was the positive staining of osteoclasts. The OPG-BMSCs group showed less osteoclasts than BMSCs and HA control group. Active resorption areas were mainly 
found in between the calcified bone and the HA scaffold, which exhibited as resorption lacunas with osteoclasts residing inside (Figs. 7A, B, C).

IHC staining of human OPG 6 weeks post-implantation was shown in Fig. S4. The OPG-BMSCs/HA group showed the strongest positive staining (yellowish color) among the three groups at 6 weeks. The BMSCs/HA group showed light positive staining, while the control group had no visible positive staining. These results confirmed the direct participation of the delivered OPG-BMSCs cells in bone regeneration.

The result of mineralized new bone area fraction was plotted in Fig. 7D. The amount of mineralized new bone area increased significantly from 4 weeks to 8 weeks in all groups $(p<$ 0.05). The OPG-BMSCs group exhibited the highest amount of mineralized new bone at each time period while the control group had the least amount of new bone $(p<0.05)$.

The number of osteoclasts was maintained at a high level in BMSCs group and the control group from 4 to 8 weeks $(p>0.05)($ Fig. 7E). The OPG-BMSCs group exhibited a significant lower number of osteoclasts than the other two groups $(p<0.05)$.

\section{Discussion}

The OVX rat model is a recommended animal model to study osteoporosis related bone diseases owing to its resemblance to the pathological changes in postmenopausal osteoporosis [23,24]. It is reported that estrogen deficiency in OVX rats can increase RANKL level $[25,26]$, enhance bone turnover and decrease bone mineral density, volume and strength [26]. The critical-sized mandibular bone defect provides an appropriate analog to 
the common clinical scenario in oral and maxillofacial surgery. Furthermore, human OPG shares $85 \%$ and $86 \%$ amino acid identity to mouse and rat OPG, respectively, indicating that the OPG has been highly conserved throughout evolution [4]. In addition, previous studies reported that the xenogeneic human OPG exerted positive effects in both mice and rats [29, 30]. To our knowledge, this is the first report of reconstruction of a critical-sized bone defect in OVX rats using OPG-BMSCs seeded HA scaffolds.

The estrogen deficiency osteoporosis is caused by dysregulated activity between the bone-forming cells (osteoblasts) and the bone-resorbing cells (osteoclasts) [2]. In order to reverse this unbalanced condition and promote bone regeneration in osteoporotic patients, we propose the novel strategy of utilizing OPG-BMSCs with the aim to inhibit bone resorption and enhance bone formation synergistically. OPG is selected as the therapeutic target for its well-known role in the RANK/RANKL/OPG pathway [5-10]. OPG acts as a decoy receptor, binds to RANKL and blocks its interaction with RANK, thus inhibits osteoclast differentiation, activation and survival [3]. The effectiveness of OPG in treatment of post-menopausal osteoporosis was first evidenced in 2001, in which the administration of OPG $\mathrm{IgG}-\mathrm{Fc}$ fusion protein $(\mathrm{OPG}-\mathrm{Fc})$ improved trabecular structure and bone strength in OVX rats, and the effects could be boosted by a combination therapy of OPG and PTH [27]. A clinical trail confirmed that blocking the RANKL-RANK system by recombinant OPG-Fc was effective in treatment of post-menopausal osteoporosis [28]. It has been reported that a single IV injection of adeno-associated virus vector carrying cDNA for recombinant human OPG was able to reverse established osteopenia in OVX mice [29]. Local administration of 
adenoviral vector-mediated OPG could also enhance osseointegration around titanium implant in OVX rats [30]. To attain a therapeutic concentration of OPG and to achieve a long-term therapeutic effect, BMSCs were used as a gene delivery vehicle in this study. Our in vitro experiments showed that the transfected BMSCs maintained a high expression of OPG gene for 21 days and OPG protein for 14 days. The in vitro bone resorption experiment showed that OPG, secreted by OPG-BMSCs, was able to suppress the osteoclast-mediated bone resorption. Thus, BMSC-mediated OPG expression was stable and functional. One study showed that systematic administration of OPG over-expressing BMSCs in myeloma bone disease mice, reduced osteoclast activation and trabecular bone loss in the vertebrae and tibiae [31]. In another study, OPG over-expressing BMSCs inhibited bone metastasis of prostate cancer by formation of new bone around tumor cells and by inhibiting osteoclast activation [7]. Our study is the first report of applying BMSCs that over-express OPG in bone tissue regeneration in osteoporotic animals. Besides anti-osteoclastogenic effect of OPG, BMSCs could also contribute to the new bone formation via direct participation or paracrine stimulation of host cells [32,33]. Animal studies indicated that transplantation of autologous or allogeneic MSCs was able to strengthen the osteoporotic bone in MSC-transplanted animals [4,35]. Thus, our strategy has two beneficial effects: exerting inhibitory effects on osteoclastogenesis via OPG and promoting bone formation via BMSCs.

In addition, it has been reported that OPG is a pro-osteogenic factor that can prime undifferentiated MSCs for the enhancement of subsequent osteogenesis [36]. Treatment of 
human MSCs with OPG before osteogenic induction significantly increased osteocalcin gene expression and mineralization, compared to untreated control cells [36]. In another study, the preosteoblast cell line MC3T3-E1 was transfected with OPG via plasmids [37]. Smad1 and Akt1, as well as ALP, were upregulated in the OPG overexpressing cells [37]. These results suggested that overexpression of OPG in preosteoblasts promoted its differentiation into mature osteoblasts [37]. The osteoblastogenesis effect could also be possible in the current study, which acts together with the aforementioned osteoclast inhibitory effect to contribute to the enhanced new bone formation in the OPG-BMSC groups.

In this study, gene therapy offers the prospect of sustained and local synthesis of OPG in situ through ex vivo gene delivery into BMSCs. Although initially used as a means of correcting defective genes, gene therapy has been applied to treating a wide range of diseases, with gene transfer being used as a type of drug-delivery system. It was reported that till 2012, over 1800 gene therapy clinical trials had been completed, were ongoing or had been approved worldwide [38]. The majority of gene therapy clinical trials to date have been aimed at treating cancer ( $64.4 \%$ of all gene therapy trials), followed by inherited monogenic diseases (8.7\%) and cardiovascular disease (8.4\%). [38]. For its use in musculoskeletal repair, even though it was well supported by preclinical animal studies, only four clinical trials for treatment of osteoarthritis and cartilage repair have taken place in the USA and Korea [39]. No clinical trial has used gene transfer to promote bone healing [39]. The lack of the translation in this field does not mean that gene therapy is ineffective. It is mainly constrained by biological safety concerns and social, financial and other factors. 
The potential insertional mutagenesis and immune responses to the vector can be lethal, even though it has only been reported under rare conditions [40-41]. As most musculoskeletal injuries are not life-threatening, the relatively high risk-to-benefit ratio hinders clinical application of gene transfer; the risk-to-benefit ratio is also important in the approval process of the regulatory bodies [39]. Nevertheless, gene therapy is still highly promising to enter this huge medical market in the future.

HA has been wildly used in bone regeneration for its excellent osteoconduction and good biocompatibility [42]. Our study showed good cell attachment and cell proliferation of both OPG-BMSCs and BMSCs on HA scaffolds. A previous study reported that the bone response of osteoporotic rats to HA was slower than that of normal rats, mainly due to highly activated osteoclasts and low level of MSCs in osteoporotic condition [43]. Thus, extended observation should be expected when HA is applied in patients with osteoporosis. In order to boost the regeneration process under this condition, combined treatments with bisphosphonate, bone forming growth factors or BMSCs have been reported [33, 44 46]. Our in vivo study showed that OPG-BMSCs groups had the best bone formation, followed by BMSCs group while the pure HA group exhibited the lowest amount of new bone formation. OPG-BMSCs were effective in inhibiting osteoclastogenesis in vitro and in vivo. These results confirmed the positive role of OPG and BMSCs in balancing bone turnover and promoting bone formation in OVX bone defect models [35, 46].

To note, BMD value of the control decreased at 8 weeks most likely due to high bone turnover in the OVX rats. Highly activated osteoclasts absorbed more HA scaffolds than 
newly deposited new bone matrices in the control group from 6 to 8 weeks. Addition of BMSCs and OPG modified BMSCs could contribute to more new bone formation, while inhibiting bone resorption via OPG.

\section{Conclusions}

In this study, we developed a novel therapeutic strategy by genetically modifying BMSCs for OPG delivery, and by seeding OPG gene-modified BMSCs on HA scaffolds to promote bone regeneration in critical-sized mandibular defects in OVX-induced osteoporotic rats. OPG genes were successfully transfected into rat BMSCs, and the OPG secreted from BMSCs was able to suppress osteoclast-mediated bone resorption in vitro. In vivo bone formation was boosted and osteoclastogenesis was reduced in defects implanted with OPG-BMSCs/HA constructs, compared to the other groups. In conclusion, the novel OPG-BMSCs/HA constructs were able to orchestrate bone-forming BMSCs and bone-resorbing osteoclasts, demonstrating good potential for osteoporotic-related bone defect reconstruction.

\section{Acknowledgements}

This work was supported by grants from the Key Technology Development Science and the Technology Department of Sichuan Province (2015SZ0127), the National Science Foundation of China (31400829) (XL) and (81401794) (PW), and the University of Maryland School of Dentistry. The funding sources have no involvement in study design, data 
collection, analysis and interpretation, writing and submission of the article.

\section{Figure captions}

1. The Ad5-OPG transfected rat BMSCs in vitro. (A) The third generation of rat BMSCs. (B) The OPG-BMSCs expressed enhanced green fluorescent protein. (C) Western blot of OPG protein in OPG-BMSCs, Ad-BMSCs and BMSCs. The OPG-BMSCs expressed hOPG protein at 14 days. (D) qRT-PCR of OPG-BMSCs, Ad-BMSCs and BMSCs. (E) ELISA assay of OPG secretion from OPG-BMSCs and Ad-BMSCs into the culture supernatants. (F) The Alizarin red staining of osteogenically differentiated OPG-BMSCs.

2. The OPG-BMSCs secreted OPG reduced bone resorption in vitro. (A) The Raw264.7 cells. (B) Raw264.7 cells were induced into multi-nucleolus osteoclast cells (black arrow show). (C) Less absorbed lacuna was formed in the Raw264.7 seeded bone wafers when co-cultured with OPG-BMSCs. (D) More mature osteoclasts and absorbed lacuna were observed when co-cultured with Ad-BMSCs. The red arrows showed the osteoclasts in the lacuna. (E) High magnification of the osteoclast in the absorbed lacuna. (F) Osteoclast density was significantly decreased when co-cultured with 
OPG-BMSCs.

3. BMSCs and OPG-BMSCs seeded on porous HA scaffolds. SEM showed that both cells well attached onto the scaffolds at day 7 (A, B). The red arrows indicated the cellular extensions into the pores of HA. (C) There was no significant differences of cell viability between the two groups $(p>0.05)$. (D) The HA scaffold was planted in the critical sized bone defect in mandible (dotted black circle).

4. Micro-CT analysis of the repaired bone defects at 4, 6 and 8 weeks. (A) Representative sagittal micro-CT images of each group at different time point. The red line dotted the region of interest area. Quantitative analysis of MV/TV ratio in the repaired bone defect area $(B)$, and BMD $(C)$. Each value is mean $\pm s d(n=6)$. Dissimilar letters indicate significantly different values $(\mathrm{p}<0.05)$.

5. Representative Masson staining images at 8 weeks. The white line dotted the defect area. More new bone was found within the defect area (red arrow) in the cell-seeded groups than the control group.

6. Representative Masson staining images at 8 weeks. Osteoclasts were found around the newly formed bone and scaffolds in the control group and BMSCs group. The OPG-BMSCs group presented the highest amount of mineralized new bone with the least amount of osteoclasts.

7. The TRAP staining results at 6 weeks (A-C) and histomorphometry analysis of (D) percentage of new bone area, and (E) osteoclast density. The red staining was the positive TRAP staining of osteoclasts in the defect area. The OPG-BMSCs group 
exhibited the highest amount of new bone at each time period but the lowest amount of osteoclasts while the control group had the lowest amount of new bone and the highest amount of osteoclasts. Each value is mean $\pm \mathrm{sd}(\mathrm{n}=6)$. Dissimilar letters indicate significantly different values $(\mathrm{p}<0.05)$.

\section{Reference}

[1] W.A. Peck, P. Burckhardt, C. Christiansen, H.A. Fleisch, H.K. Genant, C. Gennari, T.J. Martin, L. Martini, R. Morita, E. Ogata, Consensus development conference: diagnosis, prophylaxis, and treatment of osteoporosis, Am. J. Med. 94 (1993) 646-650.

[2] A.E. Kearns, S. Khosla, P.J. Kostenuik, Receptor activator of nuclear factor kappa B ligand and osteoprotegerin regulation of bone remodeling in health and disease, Endocr. Rev. 29 (2008) 155-192.

[3] P.J. Kostenuik, V. Shalhoub, Osteoprotegerin: a physiological and pharmacological inhibitor of bone resorption, Curr. Pharm. Design. 7 (2001) 613-635.

[4] W.S. Simonet, D.L. Lacey, C.R. Dunstan, M. Kelley, M.S. Chang, R. Lüthy, H.Q. Nguyen, 
S. Wooden, L. Bennett, T. Boone, G. Shimamoto, M. DeRose, R. Elliott, A. Colombero, H.L. Tan, G. Trail, J. Sullivan, E. Davy, N. Bucay, L. Renshaw-Gegg, T.M. Hughes, D. Hill, W. Pattison, P. Campbell, S. Sander, G. Van, J. Tarpley, P. Derby, R. Lee, W.J. Boyle, Osteoprotegerin: a novel secreted protein involved in the regulation of bone density, Cell. 89 (1997) 309-319.

[5] M.S. Ominsky, X. Li, F.J. Asuncion, M. Barrero, K.S. Warmington, D. Dwyer, M. Stolina, Z. Geng, M. Grisanti, H.L. Tan, RANKL inhibition with osteoprotegerin increases bone strength by improving cortical and trabecular bone architecture in ovariectomized rats, $\mathrm{J}$. Bone. Miner. Res. 23 (2008) 672-82.

[6] H. Kanzaki, M. Chiba, I. Takahashi, N. Haruyama, M. Nishimura, H. Mitani, Local OPG gene transfer to periodontal tissue inhibits orthodontic tooth movement, J. Dent. Res. 83 (2004) 920-925.

[7] D. Chanda, T. Isayeva, S. Kumar, J.A. Hensel, A. Sawant, G. Ramaswamy, G.P. Siegal, M.S. Beatty, S. Ponnazhagan, Therapeutic Potential of Adult Bone Marrow-Derived Mesenchymal Stem Cells in Prostate Cancer Bone Metastasis, Clin. Cancer. Res. 15 (2009) 7175-7185.

[8] N. Bucay, I. Sarosi, C.R. Dunstan, S. Morony, J. Tarpley, C. Capparelli, S. Scully, H.L. Tan, W. Xu, D.L. Lacey, Osteoprotegerin-deficient mice develop early onset osteoporosis and arterial calcification, Gene. Dev. 12 (1998) 1260-1268.

[9] C. Capparelli, S. Morony, K. Warmington, S. Adamu, D. Lacey, C.R. Dunstan, B. Stouch, S. Martin, P.J. Kostenuik, Sustained antiresorptive effects after a single treatment with 
human recombinant osteoprotegerin (OPG): a pharmacodynamic and pharmacokinetic analysis in rats, J. Bone. Miner. Res. 18 (2003) 852-858.

[10] B. Bolon, C. Carter, M. Daris, S. Morony, C. Capparelli, A. Hsieh, M. Mao, P. Kostenuik, C.R. Dunstan, D.L. Lacey, Adenoviral delivery of osteoprotegerin ameliorates bone resorption in a mouse ovariectomy model of osteoporosi, Mol. Ther. 3 (2001) 197-205.

[11] D.G. Phinney, D.J. Prockop, Concise review: mesenchymal stem/multipotent stromal cells: the state of transdifferentiation and modes of tissue repair-current views, Stem. Cells. 25 (2007) 2896-902.

[12] J.M. Mason, A.S. Breitbart, M. Barcia, D. Porti, R.G. Pergolizzi, D.A. Grande, Cartilage and bone regeneration using gene-enhanced tissue engineering, Clin. Orthop. Relat. R. 379 (2000) S171-S178.

[13] X. Jiang, S. Zou, B. Ye, S. Zhu, Y. Liu, J. Hu, bFGF-Modified BMMSCs enhance bone regeneration following distraction osteogenesis in rabbits, Bone. 46 (2010) 1156-1161.

[14] X. Liu, C. Bao, J. Hu, G. Yin, E. Luo, Effects of clodronate combined with hydroxyapatite on multi-directional differentiation of mesenchymal stromal cells, Arch. Med. Sci. 6 (2010) 670-677.

[15] J. Liu, W. Chen, Z. Zhao, Xu HH. Reprogramming of mesenchymal stem cells derived from iPSCs seeded on biofunctionalized calcium phosphate scaffold for bone engineering, Biomaterials. 34 (2013) 7862-7872.

[16] N. Suzuki, Y. Yoshimura, Y. Deyama, K. Suzuki, Y. Kitagawa, Mechanical stress directly suppresses osteoclast differentiation in RAW264. 7 cells, Int. J. Mol. Med. 21 (2008) 
291-296.

[17] Y. Xu, C. Guo, Y. Yan, Y. Guo, R. Li, M. Song, X. Zhang, Differential effects of mechanical strain on osteoclastogenesis and osteoclast-related gene expression in RAW264. 7 cells, Mol. Med. Rep. 6 (2012) 409-415.

[18] P. Valverde, Q. Tu, J. Chen, BSP and RANKL induce osteoclastogenesis and bone resorption synergistically. J. Bone. Miner. Res. 20 (2005) 1669-1679.

[19] Y. Gao, E. Luo, J. Hu, J. Xue, S. Zhu, J. Li, Effect of combined local treatment with zoledronic acid and basic fibroblast growth factor on implant fixation in ovariectomized rats, Bone. 44 (2009) 225-232.

[20] J. Schortinghuis, J.L. Ruben, G.M. Raghoebar, B. Stegenga, Ultrasound to stimulate mandibular bone defect healing: a placebo-controlled single-blind study in rats, J. Oral. Maxil. Surg. 62 (2004) 194-201.

[21] G Yin, J Chen, S Wei, H Wang, Q Chen, Y Lin, J Hu, and E Luo. Adenoviral vector-mediated overexpression of osteoprotegerin accelerates osteointegration of titanium implants in ovariectomized rats, Gene. Therapy. 22 (2015) 636-64.

[22] S.H. Nam, J.H. Jeong, X. Che, K.E. Lim, H. Nam, J.S. Park, J.Y. Choi, Topically administered Risedronate shows powerful anti-osteoporosis effect in ovariectomized mouse model, Bone. 50(2012) 149-155.

[23] D. Dempster, R. Birchman, R. Xu, R. Lindsay, V. Shen, Temporal changes in cancellous bone structure of rats immediately after ovariectomy, Bone. 16 (1995) 157-161.

[24] M. Yamazaki, T. Shirota, Y. Tokugawa, M. Motohashi, K. Ohno, K. Michi, A. 
Yamaguchi, Bone reactions to titanium screw implants in ovariectomized animals, Oral. Surg. Oral. Med. O. 87 (1999) 411-418.

[25] F.S. Wang, J.Y. Ko, C.L. Lin, H.L. Wu, H.J. Ke, P.J. Tai, Knocking down dickkopf-1 alleviates estrogen deficiency induction of bone loss, A histomorphological study in ovariectomized rats, Bone. 40 (2007) 485-492.

[26] X. Li, F. Asuncion, M.S. Ominsky, S. Adamu, D. Dwyer, M. Stolina, H.L. Tan, M. Grisanti, K.S. Warmington, P.J. Kostenuik, editors. RANKL inhibition with OPG preserves bone density and trabecular architecture in ovariectomized rats, J. Bone. Miner. Res. 23 (2008) 672-682

[27] P.J. Kostenuik, C. Capparelli, S. Morony, S. Adamu, G. Shimamoto, V. Shen, D.L. Lacey, C.R. Dunstan, OPG and PTH-(1-34) have additive effects on bone density and mechanical strength in osteopenic ovariectomized rats, Endocrinology. 142 (2001) 4295-4304.

[28] P.J. Bekker, D. Holloway, A. Nakanishi, M. Arrighi, P.T. Leese, C.R. Dunstan, The effect of a single dose of osteoprotegerin in postmenopausal women, J. Bone. Miner. Res. 16 (2001) 348-360.

[29] P.J. Kostenuik, B. Bolon, S. Morony, M. Daris, Z. Geng, C. Carter, J. Sheng, Gene therapy with human recombinant osteoprotegerin reverses established osteopenia in ovariectomized mice, Bone. 34 (2004) 656-664.

[30] G. Yin, J. Chen, S. Wei, H. Wang, Q. Chen, Y. Lin, J. Hu, E. Luo, Adenoviral vector-mediated overexpression of osteoprotegerin accelerates osteointegration of 
titanium implants in ovariectomized rats, Gene. Ther. 22 (2015) 636-644.

[31] N. Rabin, C. Kyriakou, L. Coulton, O. Gallagher, C. Buckle, R. Benjamin, N. Singh, J. Glassford, T. Otsuki, A. Nathwani, A new xenograft model of myeloma bone disease demonstrating the efficacy of human mesenchymal stem cells expressing osteoprotegerin by lentiviral gene transfer, Leukemia. 21 (2007) 2181-2191.

[32] X.Y. Bak, H.L. Dang, J. Yang, K. Ye, E.X. Lee, S.K. Lim, S. Wang, Human embryonic stem cell-derived mesenchymal stem cells as cellular delivery vehicles for prodrug gene therapy of glioblastoma, Hum. Gene. Ther. 22 (2011) 1365-1377.

[33] C. Evans, Gene delivery to bone, Adv. Drug. Deliv. Rev. 64 (2012) 1331-1340.

[34] Z. Wang, J. Goh, S.D. De, Z. Ge, H. Ouyang, J.S.W. Chong, S.L. Low, E.H. Lee, Efficacy of bone marrow-derived stem cells in strengthening osteoporotic bone in a rabbit model, Tissue. Eng. 12 (2006) 1753-1761.

[35] N.d.M. Ocarino, J.N. Boeloni, V. Jorgetti, D.A. Gomes, A.M. Goes, R. Serakides, Intra-bone marrow injection of mesenchymal stem cells improves the femur bone mass of osteoporotic female rats, Connect. Tissue. Res. 51 (2010) 426-433.

[36] S. Palumbo, W.J. Li, Osteoprotegerin enhances osteogenesis of human mesenchymal stem cells, Tissue. Eng. Part A. 19 (2013) 2176-2187.

[37] H.Y. Y, P. Vos, Y.J. Ren, Overexpression of osteoprotegerin promotes preosteoblast differentiation to mature osteoblasts, Angle. Orthod. 81 (2011) 100-106.

[38] S.L. Ginn, I.E. Alexander, M.L. Edelstein, M.R. Abedi, J. Wixon, Gene therapy clinical trials worldwide to 2012-an update, J. Gene. Med 15 (2013) 65-77. 
[39] C.H. Evans, J. Huard, Gene therapy approaches to regenerating the musculoskeletal system, Nat. Revi. Rheumato. 11 (2015) 234-242.

[40] C. Hacein-Bey-Abina, C VonKalle, M. Schmidt, M.P. Mccormack, N. Wulffraat, LMO2-associated clonal $\mathrm{T}$ cell proliferation in two patients after gene therapy for SCID-X1, Science. 302 (2003) 415-419.

[41] C.S. Manno, V.R. Arruda, G.F. Pierce, B. Glader, M. Ragni, J. Rasko, M.C. Ozelo, K. Hoots, P. Blatt, B. Konkle, M. Dake, R. Kaye, M. Razavi, A. Zajko, J. Zehnder, H. Nakai, A. Chew, D. Leonard, J.F. Wright, R.R. Lessard, J.M. Sommer, M. Tigges, D. Sabatino, A. Luk, H. Jiang, F. Mingozzi, L. Couto, H.C. Ertl, K.A. High, M.A. Kay, Successful transduction of liver in hemophilia by AAV-factor IX and limitations imposed by the host immune response, Nat. Med. 12 (2006) 342-347.

[42] R. Pilliar, M. Filiaggi, J. Wells, M. Grynpas, R. Kandel, Porous calcium polyphosphate scaffolds for bone substitute applications - in vitro characterization, Biomaterials. 22 (2001) 963-972.

[43] T. Saito, Y. Kin, T. Koshino, Osteogenic response of hydroxyapatite cement implanted into the femur of rats with experimentally induced osteoporosis, Biomaterials. 23 (2002) 2711-2716.

[44] Y. Li, E. Luo, G. Feng, S. Zhu, J. Li, J. Hu, Systemic treatment with strontium ranelate promotes tibial fracture healing in ovariectomized rats, Osteoporosis. Int. 21 (2010) 1889-1897.

[45] G. Turgeman, Y. Zilberman, S. Zhou, P. Kelly, I.K. Moutsatsos, Y.P. Kharode, L.E. 
Borella, F.J. Bex, B.S. Komm, P.V. Bodine, Systemically administered rhBMP-2 promotes MSC activity and reverses bone and cartilage loss in osteopenic mice, J. Cell. Biochem. 86 (2002) 461-474.

[46] Y. Tang, W. Tang, W. Tian, X. Chen, S. Li, A study on repairing mandibular defect by means of tissue-engineering and human bone morphogenetic protein-2 gene transfection in osteoporotic rats, Zhonghua. Kou. Qiang. Yi. Xue. Za. Zhi. 41 (2006) 430-431. 


\section{Figure 1}
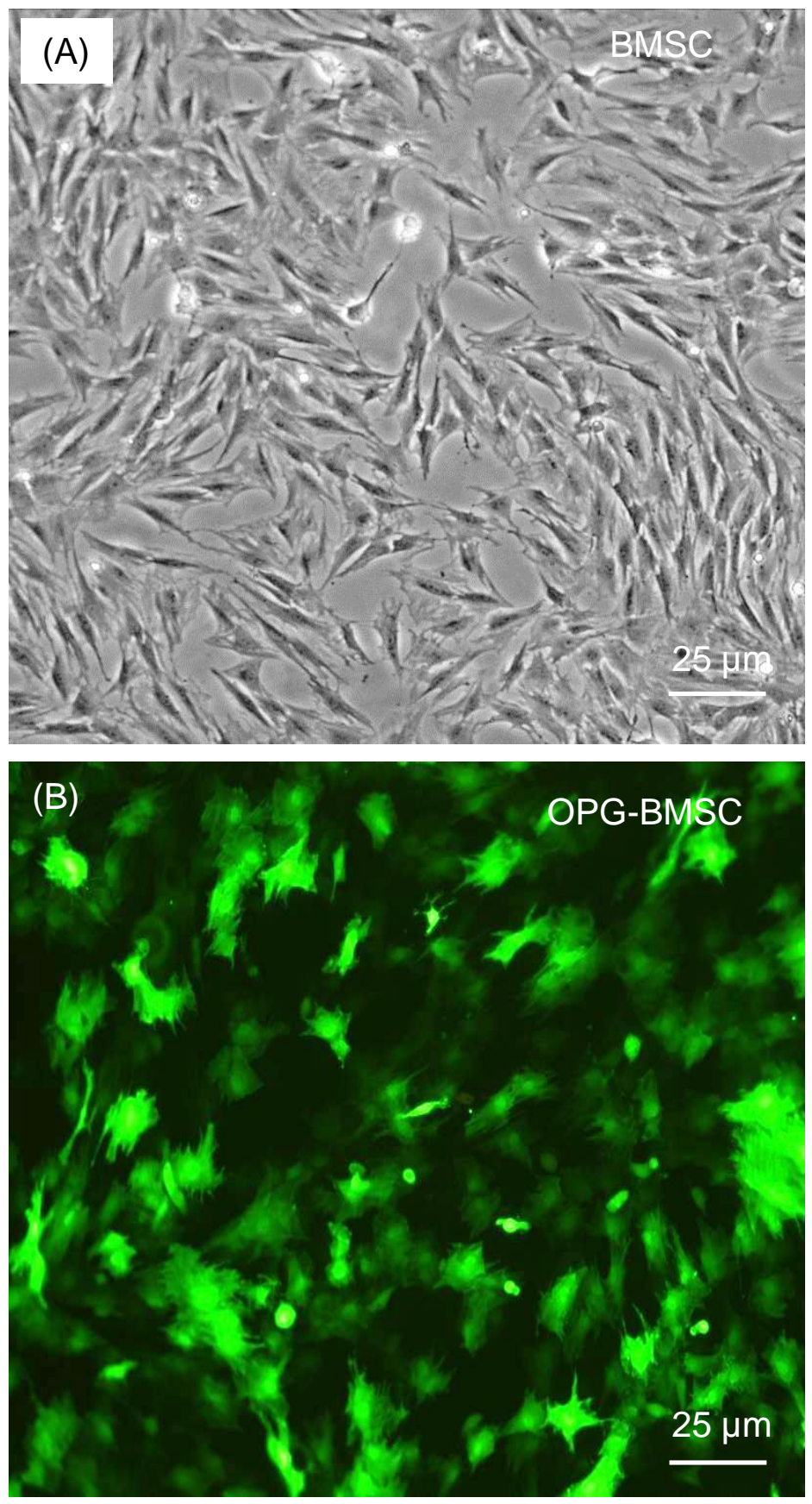

(C)
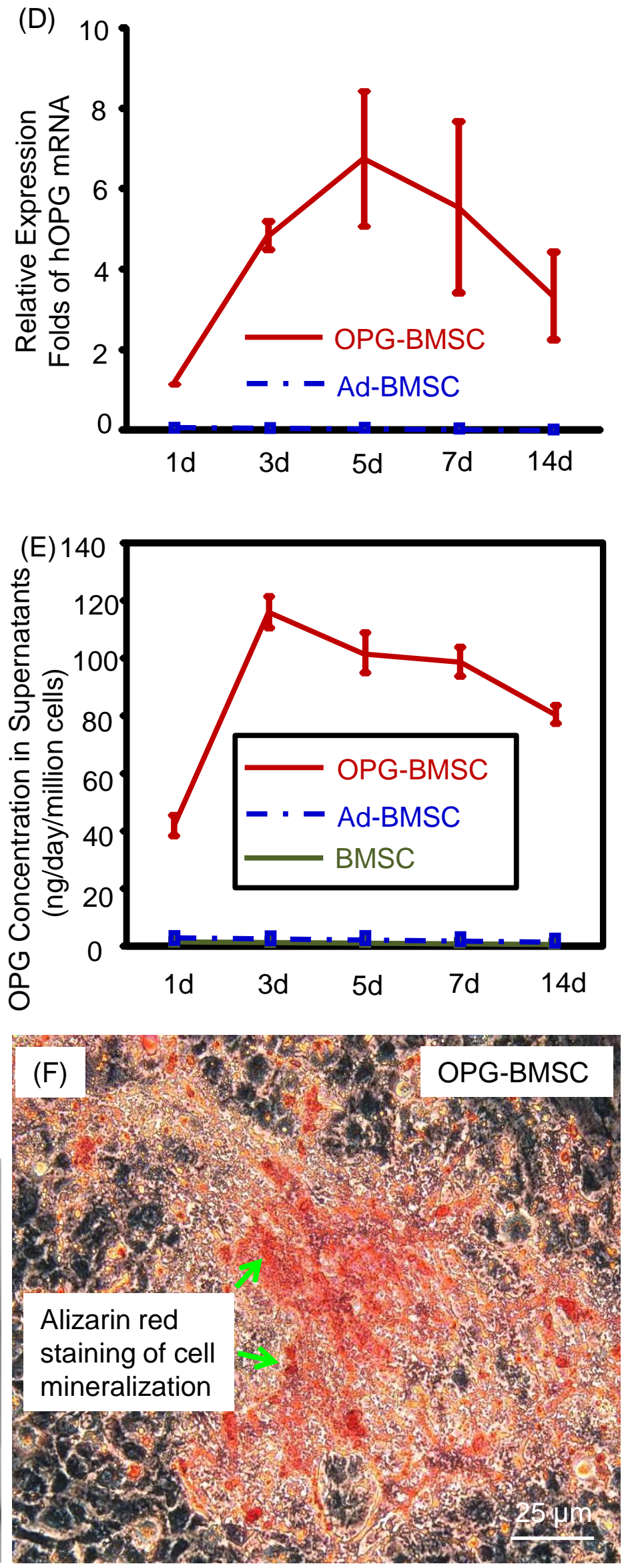

Fig. 1 

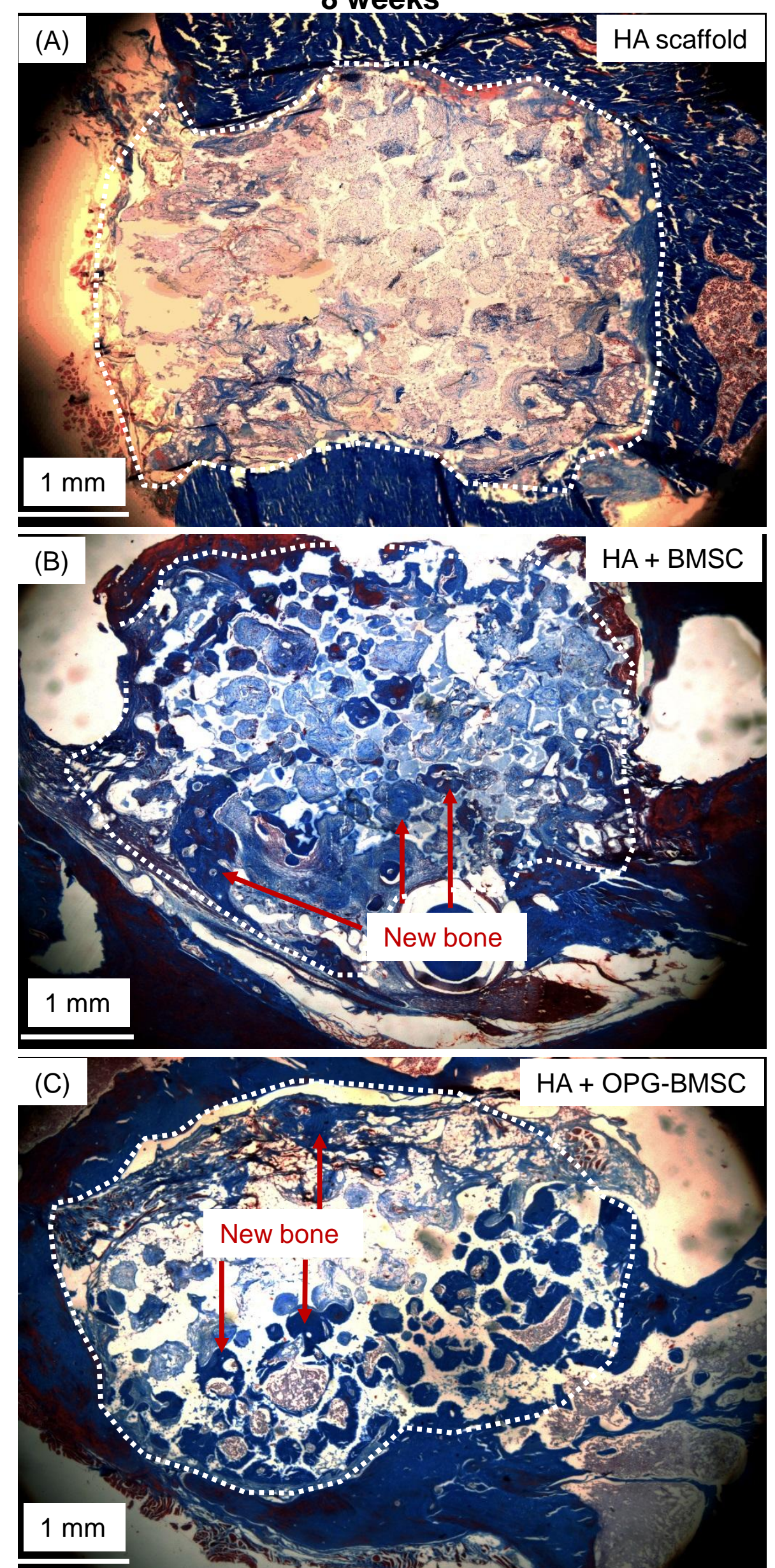
